\title{
Tracking Performance of a Single-Crystal and a Polycrystalline Diamond Pixel-Detector
}

\author{
Dario Menasce $^{b}$, Luigi Moroni ${ }^{b, *}$, Jennifer Ngadiuba ${ }^{b}$, Lorenzo Uplegger ${ }^{a}$,Enver \\ Alagoz $^{c}$, Jeff Andresen ${ }^{a}$, Kirk Arndt ${ }^{c}$, Gino Bolla ${ }^{c}$, Daniela Bortoletto ${ }^{c}$, Jean Marie \\ Brom $^{d}$, Richard Brosius ${ }^{e}$, Mayur Bubna ${ }^{c}$, John Chramowicz ${ }^{a}$, John Cumalat ${ }^{f}$, \\ Mauro Dinardo o ,Paolo Dini ${ }^{b}$,Frank Jensen ${ }^{f}$, Alex Krzywda ${ }^{c}$, Ashish Kumar ${ }^{e}$, Simon \\ $\mathrm{Kwan}^{a}{ }, \mathrm{CM}$ Lei $^{a}$, Margherita Obertino ${ }^{g}$, Ilya Osipenkov ${ }^{h}$, Lalith Perera ${ }^{i}$, Alan \\ Prosser $^{a}$, Ryan Rivera ${ }^{a}$, Ada Solano ${ }^{g}$, Ping $\operatorname{Tan}^{a}$, Silvia Tentindo ${ }^{\prime}$, Stefano Terzo ${ }^{b}$, \\ Nhan Tran and Stephen R. Wagner \\ ${ }^{a}$ Fermi National AcceleratorLaboratory, \\ P.O. Box 500, Batavia, IL 60510-0500, USA \\ ${ }^{b}$ Istituto Nazionale di Fisica Nucleare, Sezione di Milano Bicocca, and Università degli Studi di \\ Milano Bicocca, \\ Piazza della Scienza 3, 20126 Milano, Italy \\ ${ }^{c}$ Purdue University, \\ Department of Physics, 1396 Physics Building, West Lafayette, IN 47907-1396, USA \\ ${ }^{d}$ Strasbourg IPHC, Institut Pluridisciplinaire Hubert Curien, \\ 23, rue du Loess, B.P. 28, F-67037 Strasbourg Cedex, France \\ ${ }^{e}$ State University of New York at Buffalo (SUNY), \\ Department of Physics, 239 Fronczak Hall, Buffalo, NY 14260-1500, USA \\ ${ }^{f}$ University of Colorado at Boulder, \\ Department of Physics, Boulder, CO 80309 -0390, USA \\ ${ }^{g}$ Istituto Nazionale di Fisica Nucleare, Sezione di Torino, \\ Via Pietro Giuria 1, 10125 TORINO, Italy \\ ${ }^{h}$ Texas A\&M University, \\ Department of Physics, 4242 TAMU, College Station, TX 77843-4242, USA \\ ${ }^{i}$ University of Mississippi, \\ Department of Physics and Astronomy, 108 Lewis Hall, University, MS 38677, USA \\ ${ }^{j}$ Florida State University, \\ 600 W. College Avenue, Tallahassee, FL 32306, USA \\ E-mail: Luigi.Moroni@mib.infn.it
}

ABSTRACT: We present a comparative characterization of the performance of a single-crystal and a polycrystalline diamond pixel-detector employing the standard CMS pixel readout chips. Measurements were carried out at the Fermilab Test Beam Facility, FTBF, using protons of momentum $120 \mathrm{GeV} / \mathrm{c}$ tracked by a high-resolution pixel telescope. Particular attention was directed to the study of the charge-collection, the charge-sharing among adjacent pixels and the achievable position resolution. The performance of the single-crystal detector was excellent and comparable to the best available silicon pixel-detectors. The measured average detectionefficiency was near unity, $\varepsilon=0.99860 \pm 0.00006$, and the position-resolution for shared hits was

${ }^{*}$ Corresponding author. 
about $6 \mu \mathrm{m}$. On the other hand, the performance of the polycrystalline detector was hampered by its lower charge collection distance and the readout chip threshold. A new readout chip, capable of operating at much lower threshold (around $1 \mathrm{ke}^{-}$), would be required to fully exploit the potential performance of the polycrystalline diamond pixel-detector.

KEYWORDS: Diamond Detectors; Radiation-hard detectors; Solid state detectors. 


\section{Contents}

1. Introduction

2. Single-crystal diamond pixel-detector performance $\quad 2$

3. Polycrystalline diamond pixel-detector performance 6

4. Conclusions

\section{Introduction}

Diamond has been shown to be one of the most promising alternatives to silicon for applications as a sensor material for solid-state detectors in very high radiation environments [1]. In particular, it is one of the best candidates for the innermost barrel layer of the CMS pixel detectors in view of the foreseen upgrade of LHC to High-Luminosity LHC. The present generation of planar silicon pixel detectors, indeed, cannot survive the required minimal fluence of about $10^{16} \mathrm{neq} / \mathrm{cm}^{2}$ without significant degradations in the performance and, therefore, need to be replaced.

This paper investigates the charge-collection features in single-crystal (scCVD) and polycrystalline (pCVD) diamond pixel detectors. For a direct and more reliable comparison of the performance, we used the same type of CMS pixel readout chip [2] for the two detectors. Nonetheless, as discussed below, a new and much more sensitive pixel readout chip is required to fully exploit the potential of the polycrystalline diamond. The optimal design specifications for this new chip are clearly impacted by the present results.

The scCVD and pCVD detector prototypes were tested in the $120 \mathrm{GeV} / \mathrm{c}$ proton beam available at the Fermilab ${ }^{1}$ Test Beam Facility. Beam tracks were reconstructed using a telescope of 8 planes of CMS pixel detectors with pixel size of $100 \times 150 \mu \mathrm{m}^{2}$ [3]. The resolution on the transverse coordinates of the track impact-point on the detector under test was about $7 \mu \mathrm{m}$. The prototypes were placed at the center of the telescope and orthogonal to the beam axis with a better than $2^{\circ}$ accuracy. The reconstructed beam-track divergence was less than $10^{-4} \mathrm{rad}$. The telescope detectors and the prototypes were precisely aligned by means of a program developed ad-hoc by the Milano-Bicocca group, which allows for all possible degrees of freedom both on translations and rotations of each element. Data acquisition was performed using the CAPTAN system, a fast, modular, FPGA-based system developed by Fermilab, which can be easily adapted to any kind of device [4]. The charge calibration of the pixel-channel signals was accomplished using the CMS readout chip internal calibration circuitry, which injects the same amount of charge in all the selected channels. In this paper, the unit charge for calibrations is indicated by $e_{c a l}^{-}$since it should roughly correspond to unit electron charge, $e^{-}$.

\footnotetext{
${ }^{1}$ Fermi National Accelerator Laboratory
} 


\section{The single-crystal diamond pixel-detector performance}

The scCVD diamond sensor, $4.7 \times 4.7 \mathrm{~mm}^{2}$ area and $500 \mu \mathrm{m}$ thickness, produced by Element 6 , had $100 \times 150 \mu \mathrm{m}^{2}$ pixel-cells to match the CMS readout chip and covered roughly $1 / 4$ of the chip area. The pixels, and the HV pad on the opposite side of the diamond sensor, were Tungsten Titanium (W/Ti, 90\%/10\% by weight) and were sputtered on the diamond to a thickness of $100 \mathrm{~nm}$. There was $20 \mu \mathrm{m}$ between pixels on each side, so that the W/Ti metal pad covered $69 \%$ of the pixel unit cell. The diamond surfaces were first acid-cleaned to remove traces of previous metal depositions and then washed in deionized water. Then the metal was deposited, and the diamond was annealed at $400 \mathrm{C}^{\circ}$ for 4 minutes in a Nitrogen atmosphere. Finally the pixels were Indium bump-bonded onto the readout chip.

The sensor's measured CCD (Charge Collection Distance, i.e. the average distance an $e-h$ pair moves apart in a $1 \mathrm{~V} / \mu \mathrm{m}$ E-field) was $(508 \pm 50) \mu \mathrm{m}=\frac{(18.3 \pm 1.8) k e^{-}}{36 e^{-} / \mu \mathrm{m}}$, where $(18.3 \pm 1.8) k e^{-}$ is the mean signal we measured for normally incident minimum-ionizing particles and $36 e^{-} / \mu \mathrm{m}$ is the expected mean signal per micron of track length when the produced charge is fully collected [6]. The measured CCD amounts to about the thickness of diamond, meaning that all charge is collected at the electrodes as expected for single crystal detectors. The biasvoltage applied to the detector was $500 \mathrm{~V}$. The average threshold for pixel-readout was about $3.5 \mathrm{ke}_{\text {cal }}^{-}$with a $\sigma \approx 1.5 \mathrm{ke}_{\text {cal }}^{-}$dispersion. These figures are surprisingly high for this readout chip, which typically features an average threshold of $2.5 \mathrm{ke}_{\text {cal }}^{-}$with a $\sigma \approx 0.5 \mathrm{ke}_{\text {cal }}^{-}$dispersion. Nevertheless, this problem apart, the chip was perfectly working.

Fig. 1 shows the average charge collected by the pointed-to pixel only ${ }^{2}$ as a function of the track impact-point coordinates relative to the pixel center (a) and the four-pixel corner (b).
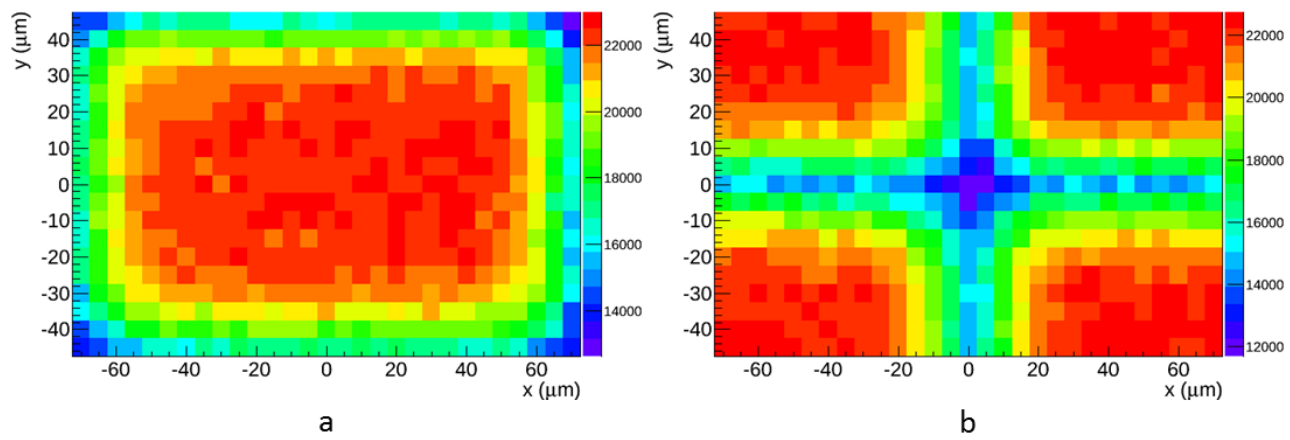

Figure 1: Average charge collected by the pointed-to pixel alone as a function of the track impact-point coordinates relative to the pixel center (a) and the four-pixel corner (b).

The collected charge is practically constant at the center of the pixel and starts to decrease from about $20 \mu \mathrm{m}$ from the edges. This loss of charge is due to the combined effect of the track impact point error, $\sigma_{\text {track }} \approx 7 \mu \mathrm{m}$ both in $x$ and $y$, and the charge-sharing with adjacent pixels.

The cluster-size distribution of the hits, i.e. the number of adjacent pixels fired by a track, is given in Fig.2. The measured percentage of single-hits (cluster-size=1) with respect to the total is $\approx 76 \%$.

\footnotetext{
${ }^{2}$ We refer to the pixel to which the track points as the pointed-to pixel.
} 


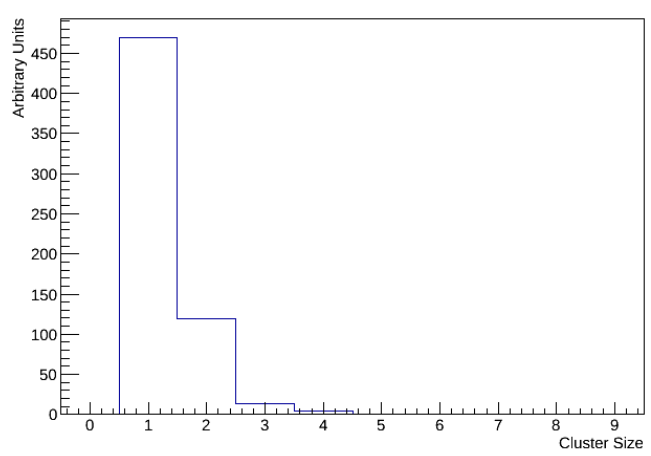

Figure 2: Cluster-size distribution of the hits.

The single-hit charge spectrum is shown in Fig.3 requiring the track impact-point to be more than $20 \mu \mathrm{m}$ away from the pixel edges on both the coordinates. This fiducial area cut was applied to avoid that the events with an undetected charge-sharing with adjacent pixels could contribute and bias the distribution.
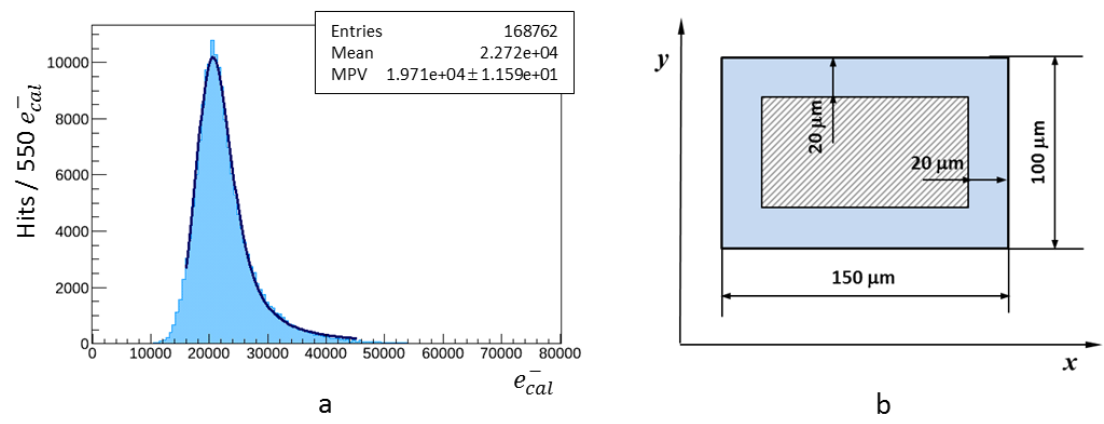

Figure 3: Single-hit charge spectrum (a) and associated fiducial area cut for tracks (b). An estimate of the actual charge in electrons can be obtained multiplying the horizontal scale of (a) by 0.81 .

The resulting Most Probable Value (MPV) [5] of the spectrum is about $19.7 \mathrm{ke}_{\text {cal }}^{-}$. The mean value, $22.7 \mathrm{ke}_{\text {cal }}^{-}$, can be directly compared with that we measured to evaluate the $\mathrm{CCD}$ of this sensor, $18.3 \pm 1.8 \mathrm{ke}^{-}$. This tells us that, in this case, the absolute scale of our calibrations should be multiplied by $0.81 \pm 0.08$ to get the right value. We do not correct for this because it wouldn't impact our results to any significant extent.

In a similar way, we measured the spectrum of double-hits (cluster-size=2) belonging to a single row or column requiring that the track impact-point be more than $20 \mu \mathrm{m}$ away from the row or column edges respectively (Fig. 4). Again, this cut prevents biasing of the distributions due to undetected additional sharing with a third pixel-cell on an adjacent row or column.

The MPV of the two spectra well matches that of the single hits as it should. Nonetheless, the distributions are widened by the sum of the uncorrelated dispersions of the two signals, which are affected by both calibration errors and electronic noise. In addition, the tails of the distributions at high values (e.g. above $30 \mathrm{ke}_{\text {cal }}^{-}$) are certainly boosted by the joint effect of threshold-cut and $\delta$-rays. 

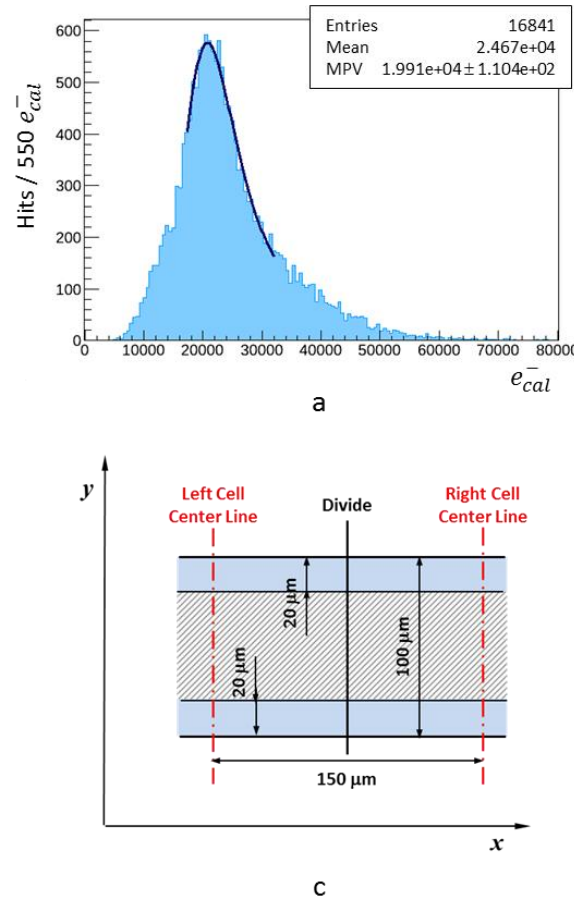
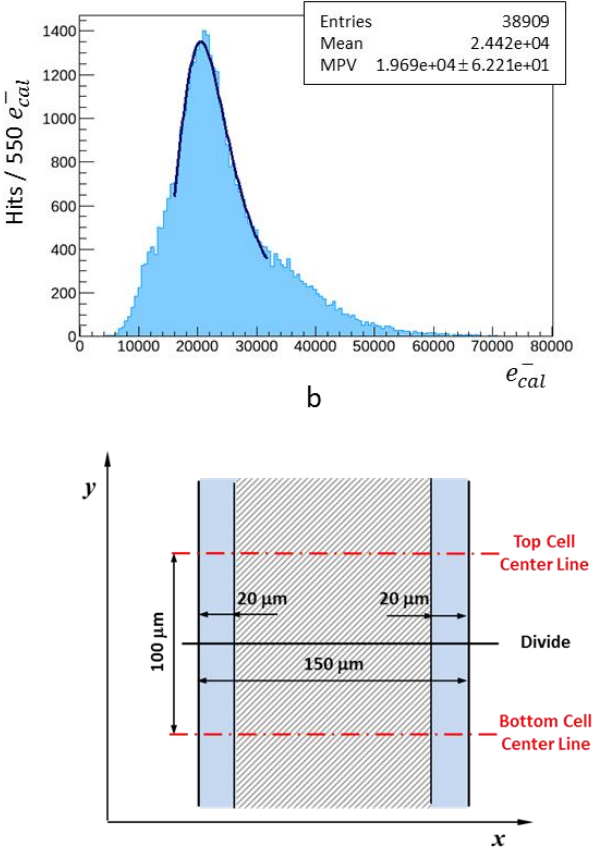

Figure 4: Spectra of double hits on a row (a), on a column (b), and associated fiducial area cuts.

With the same fiducial area cuts (see Fig.4c and 4d) we studied the charge-collection efficiency along a row or a column. The results, requiring that the pointed-to pixel is always above threshold, are shown in Fig.5 as a function of the distance of the track impact-point from the divide of two adjacent pixels on a row, Fig.5a, or on a column, Fig.5b. The black points represent the mean value of the charge collected by the pointed-to pixel, whereas the red points represent the sum of the charge from the two adjacent pixels. When the track is far away from the divide, the charge is fully collected by the pointed-to pixel and, therefore, no appreciable contribution comes from the adjacent pixel. When the track is near the divide (within the track reconstruction error), the charge starts to be shared with the adjacent pixel and, because of the threshold-cut, part of it can be lost. Anyhow, in the worst case, more than $90 \%$ of the charge can be recovered by summing the charges collected by the two adjacent pixels.
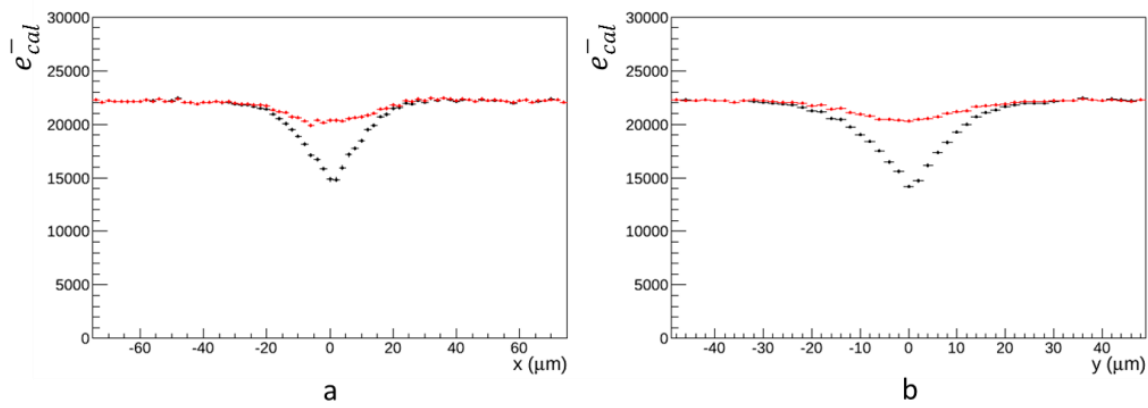

Figure 5: Mean value of the charge collected by the pointed-to pixel only (black points) and that collected together with the nearer adjacent pixel (red points) on the same row (a) and on the same column (b). For further details see text. 
The detection efficiency is presented in Fig. 6 as a function of the track impact-point coordinates within the pointed-to pixel. To avoid biases due the track impact point error, the pointed-to pixel is declared efficient if its signal, or one of the eight pixels around it, is above threshold.

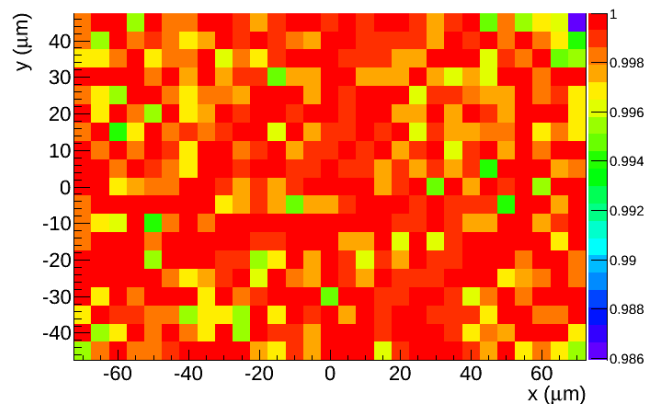

Figure 6: Detection efficiency as a function of the track impact-point within a pixel cell.

The efficiency is very high and does not show any drop even near the corners of pixel cell. Its value averaged over the cell is very close to unity, $\varepsilon=0.99860 \pm 0.00006$.

The request for efficiency evaluation can be made more selective by limiting our investigation to some specific region of the pixel cell. For instance, using the same fiducial area cuts of Fig.4, we can demand for the efficiency of the only two pixels which could be hit by the track. The resulting evolution of the detection efficiency along a row or a column is reported in Fig.7. The black points represent the efficiency of the pointed-to pixel alone, while the red points represent the joint efficiency with the nearer adjacent pixel.

The plots show that the two pixels do actually ensure full efficiency. In particular, the pointed-to pixel alone provides full efficiency when the track is far from the divide of the two adjacent pixels, and it starts to lose efficiency as soon as the track approaches the divide. This drop of efficiency is caused both by the track impact-point error and by the threshold-cut in the much narrower charge-sharing region about the divide.
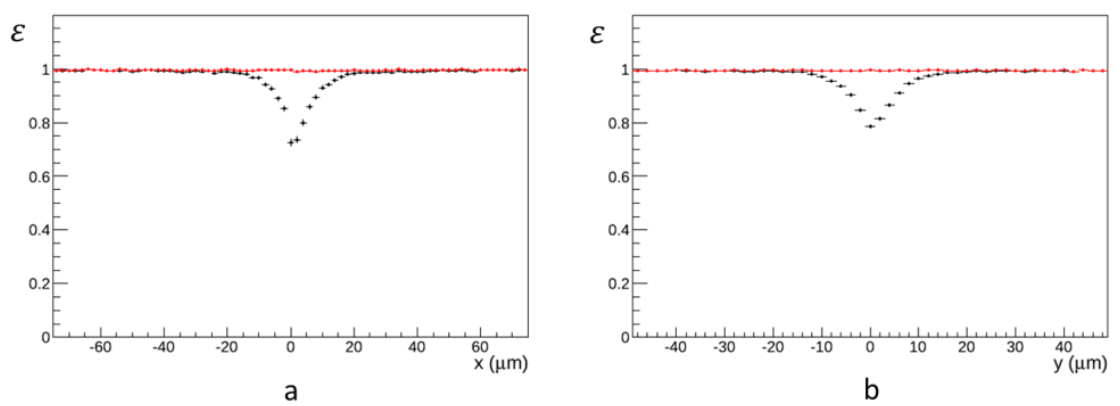

Figure 7: Detection efficiency evolution along a row (a) and a column (b); black points are for the efficiency of the pointed-to pixel alone and red points for the joint efficiency with the nearer adjacent pixel on the same row or column.

The last study we carried out to complete the characterization of the detector concerns the features of the charge division. We studied it for two adjacent hits and exploited it to improve the detector position resolution. To this extent we defined the charge asymmetry, $\eta=\frac{Q_{L}-Q_{R}}{Q_{L}+Q_{R}}$, where $Q_{L}$ is the charge collected by the pixel on the left and $Q_{R}$ that by the pixel on the right with respect to the track impact-point. We then measured the correlation of the asymmetry with the track impact-point coordinates, $x$, along the pixel rows, and $y$, along the columns. We 
applied the usual fiducial area cuts of Fig.4 to restrict the charge sharing to only two adjacent pixels and required $Q_{L}+Q_{R}<30 \mathrm{ke}_{\text {cal }}^{-}$to avoid biases from energetic $\delta$-rays.

The measured $x-\eta$ and $y-\eta$ correlations are shown in Fig. 8 both at single-event level and when the mean coordinate is calculated for each bin of $\eta$.
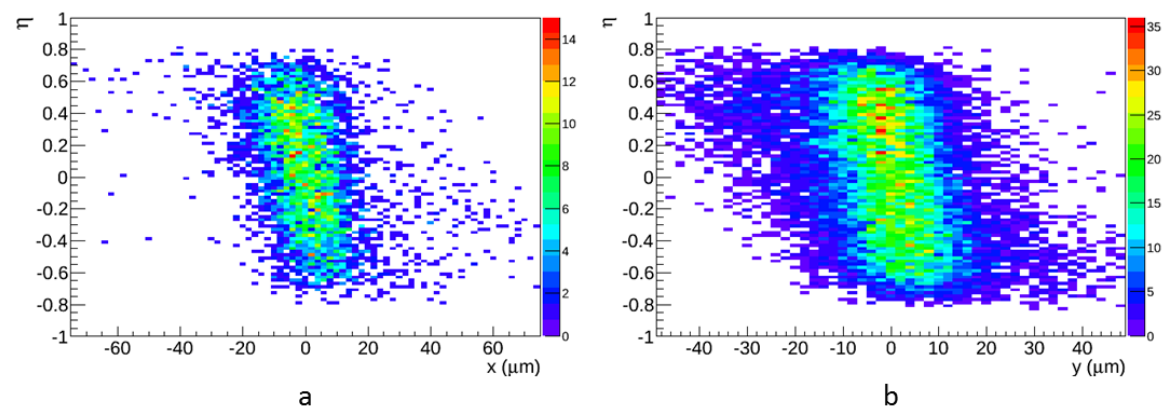

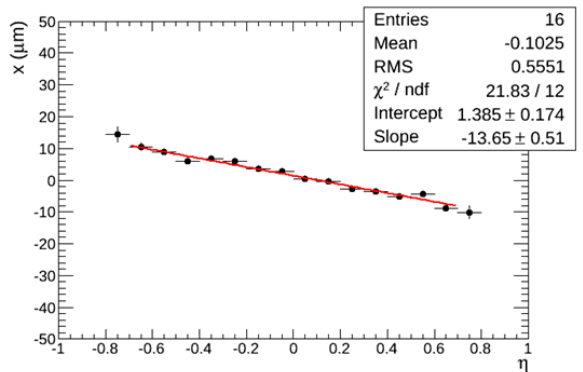

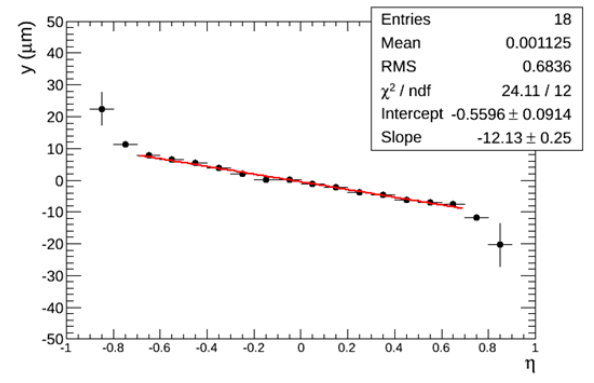

d

Figure 8: $x-\eta$ (a) and $y-\eta$ (b) correlation plots and their coordinate-averaged regressions. For further details see text.

The resulting correlations are linear, to a good approximation, up to $|\eta|=0.7$ and their fitted slopes are certainly consistent, if we take into account the orthogonality error of the detector to the beam. The deviation from the normality could indeed be different for the $x$ and $y$-coordinates of the detector.

Inverting the argument, we can therefore obtain an optimal estimate of the double-hit coordinates from the measured asymmetry values. A crude evaluation of the achievable position resolution can be obtained from the residuals with the telescope tracks. In fact, subtracting in quadrature the known track error from the residual width, we can estimate a detector positionresolution for double-hits of about $6 \mu \mathrm{m}(\sigma \approx 6 \mu \mathrm{m})$ both in $x$ and $y$.

\section{The polycrystalline diamond pixel-detector performance}

For the sake of clarity we warn the reader that we will improperly use the term signal induction to indicate the effect responsible for the difference between the signal-charge on an electrode and the charge actually collected at that electrode [7] [8]. In absence of charge trapping, as for scCVD diamond, signal induction is practically absent since it would be eventually zeroed when all the produced charge is collected at the electrodes. By way of contrast, when dealing with pCVD diamond we expect sizeable signal induction contributions: a net charge signal could be even induced on the electrodes that are not collecting charge at all. The size of signal induction would in general depend on the sensor thickness to pixel-pitch ratio and CCD. 
The polycrystalline sensor, produced by Element 6 , had a larger area, $10 \times 10 \mathrm{~mm}^{2}$, but the same thickness, $500 \mu \mathrm{m}$, as the scCVD sensor. The metallization, annealing and bump-bonding processes were identical to those for the scCVD sensor. The measured CCD was $175 \pm 18 \mu \mathrm{m}$, much smaller than its thickness, meaning that a large fraction of the charge produced by the ionizing particles gets trapped in the bulk and doesn't reach the electrodes. This is typical for a polycrystalline diamond and, more generally, for any type of sensor which has been irradiated. In other words, we can say that the performance of our pCVD diamond is very close to that expected even for a scCVD diamond once it has been exposed to a fluence of roughly $6 \times 10^{15}$ $n e q / \mathrm{cm}^{2}$ (see for instance Section 2.2 of reference [9]). Therefore, the present detector provides us with a good example of what we would eventually face operating a detector in a very harsh radiation environment.

During our tests, the bias-voltage applied to the detector was $500 \mathrm{~V}$. The average threshold for pixel-readout was about $2.5 \mathrm{ke}_{\text {cal }}^{-}$with a $\sigma \approx 0.5 \mathrm{ke} e_{\text {cal }}^{-}$dispersion.

The same set of measurements performed on the single-crystal detector was repeated with the polycrystalline diamond pixel detector. In particular, the measurement of the average charge collected by the pointed-to pixel alone as a function of the track impact-point coordinates is shown in Fig.9.
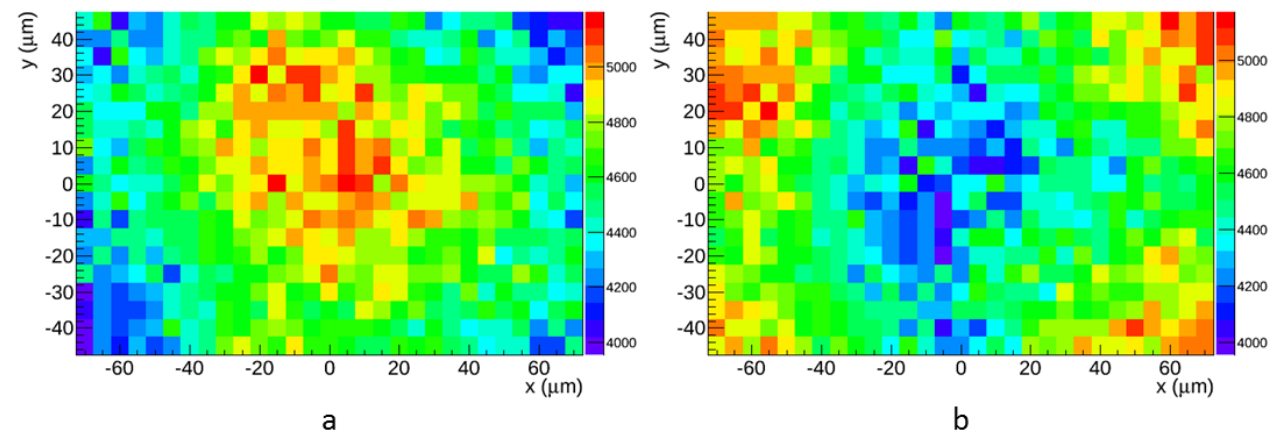

Figure 9: Average charge collected by the pointed-to pixel alone as a function of the track impact-point coordinates relative to the pixel center (a) and the four-pixel corner (b).

Charge-sharing seems to impact signal even at more than $30 \mu \mathrm{m}$ away from the pixel edges. We will see that, very likely, the actual signals are always spread over two pixels at least by signal induction.

The cluster-size distribution of the hits is given in Fig.10.

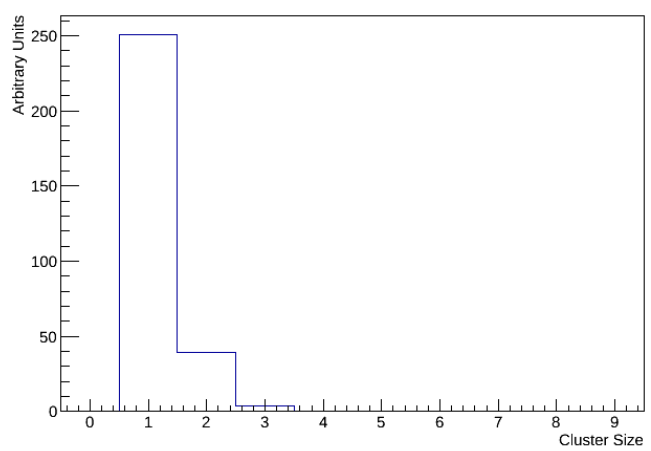

Figure 10: Cluster-size distribution of the hits. 
This time, the fraction of single-hits (cluster-size=1) turns out to be about $83 \%$, a surprisingly low percentage given the relatively much higher threshold we applied. We will see that this is due to the presence of a sizeable signal induction effect.
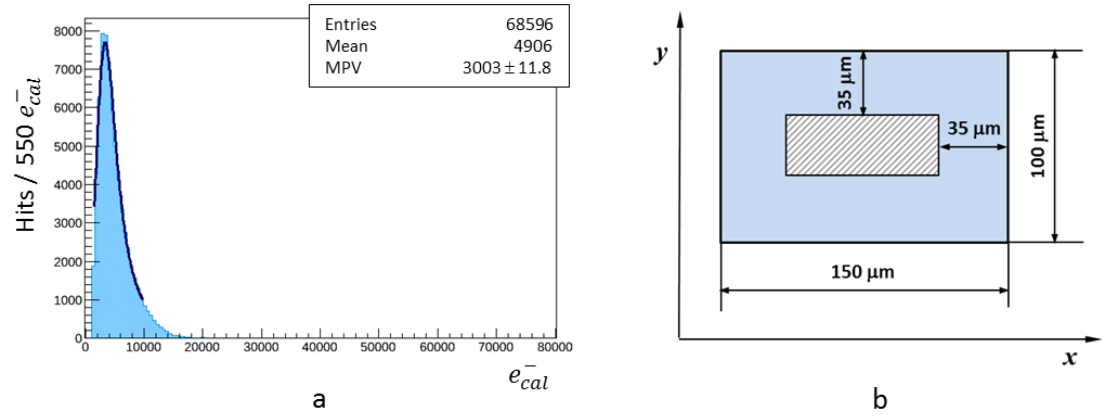

Figure 11: Single hit spectrum (a) and associated fiducial area cut for tracks (b).
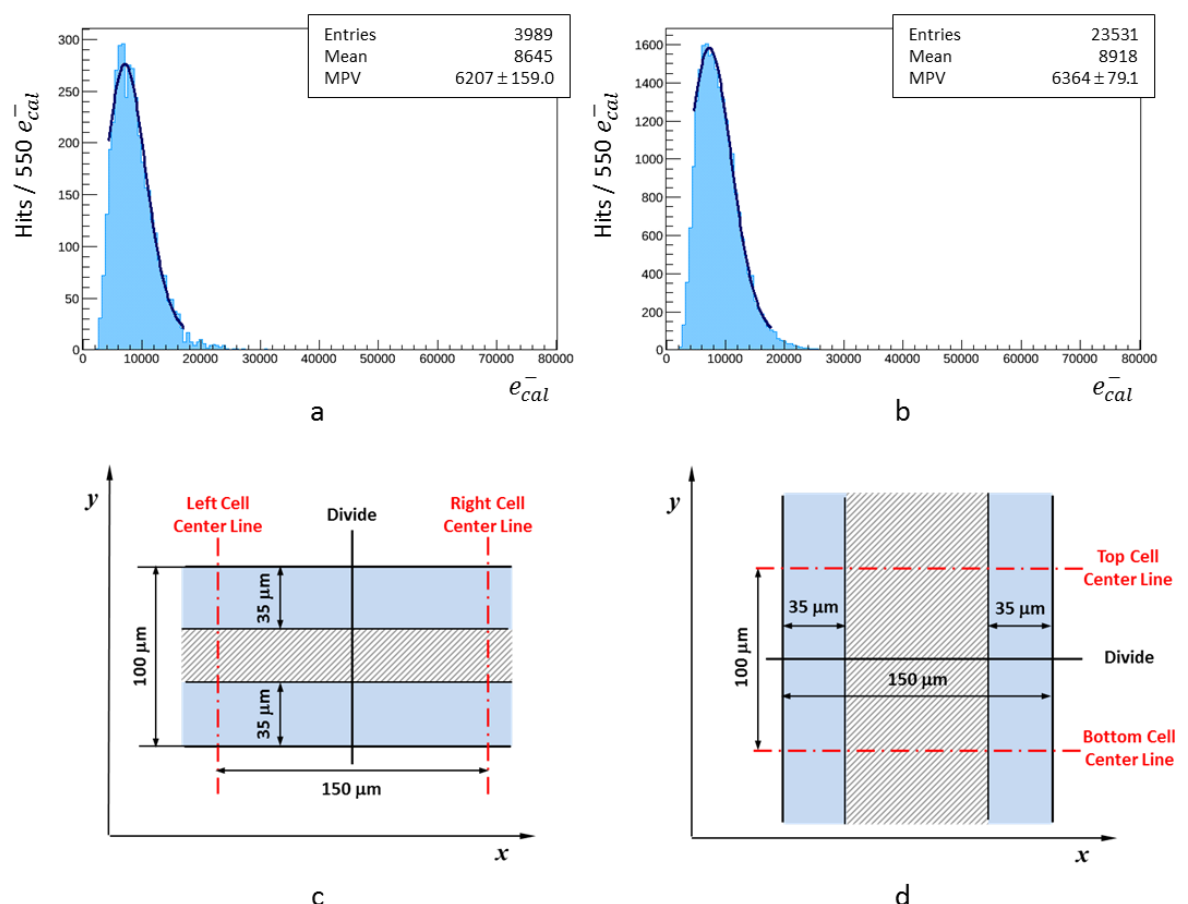

Figure 12: Spectra of double hits on a row (a), on a column (b), and associated fiducial area cuts.

Fig. 11 shows the single-hit pulse height spectrum and the associated fiducial area cut, which was further restricted to $35 \mu \mathrm{m}$ away from the edges to partially limit the charge sharing with other pixels, without running out of statistics.

The observed spectrum has lost its original Landau shape because of the applied threshold cut. Therefore we couldn't even compare the measured MPV with the expected value. Nonetheless,

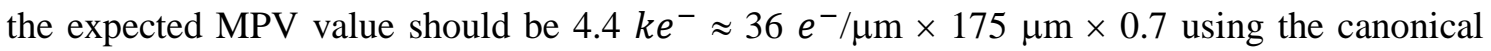


value of 0.7 for the $\frac{Q_{M P V}}{Q_{\text {mean }}}$ ratio in case of pCVD Diamond [6]. If we allow for an additional reduction of roughly $20 \%{ }^{3}$ to account for undetected signal induction on nearby pixels, we obtain $3.5 \mathrm{ke}^{-}$to be compared with a measured value of $3.0 \mathrm{ke}-\mathrm{cal}^{-}$. This suggests that the absolute scale of our calibrations shouldn't be far from the correct value.

The double-hit spectra (cluster-size=2) are reported in Fig. 12 together with the fiducial area cuts applied. As expected, the threshold effect is such to select very large charge releases in the sensor and, therefore, to push the MPV of the spectra toward high values, much higher than the single-hit MPV (6.3 $\mathrm{ke}_{\text {cal }}^{-}$versus $\left.3.0 \mathrm{ke}-{ }_{\text {cal }}^{-}\right)$. For this reason, the surviving events are heavily contaminated by $\delta$-rays.

At this point, it is very interesting to check how these features compare with our expectations and in particular with those of the scCVD detector previously tested. To this extent we made use of the scCVD data and cut them with a $15 \mathrm{ke}_{\text {cal }}^{-}$threshold, which is about $80 \%$ of single-hit peak, as in the present case. The resulting histograms, Fig.13, show that the double-hit MPV is shifted to about twice the single-hit MPV, $38 k_{\text {cal }}^{-}$versus $19 k e_{\text {cal }}^{-}$, as in our measurements.

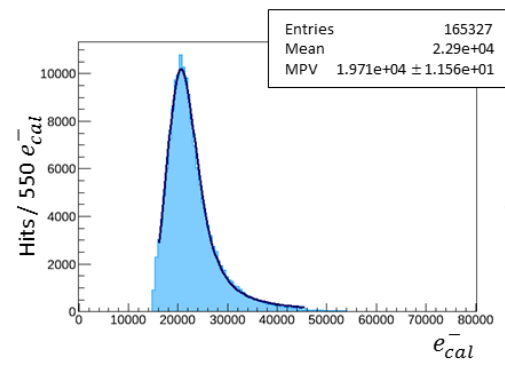

a

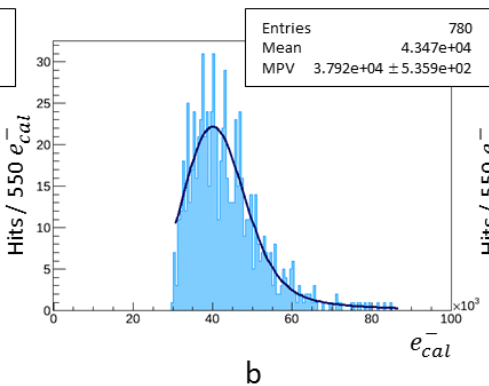

b

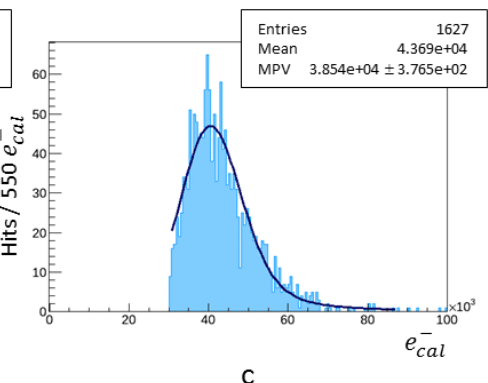

Figure 13: Spectra of single hits (a), double hits on a row (b) and double hits on a column (c) of the scCVD detector with a $15 \boldsymbol{k e}_{c a l}^{-}$threshold.
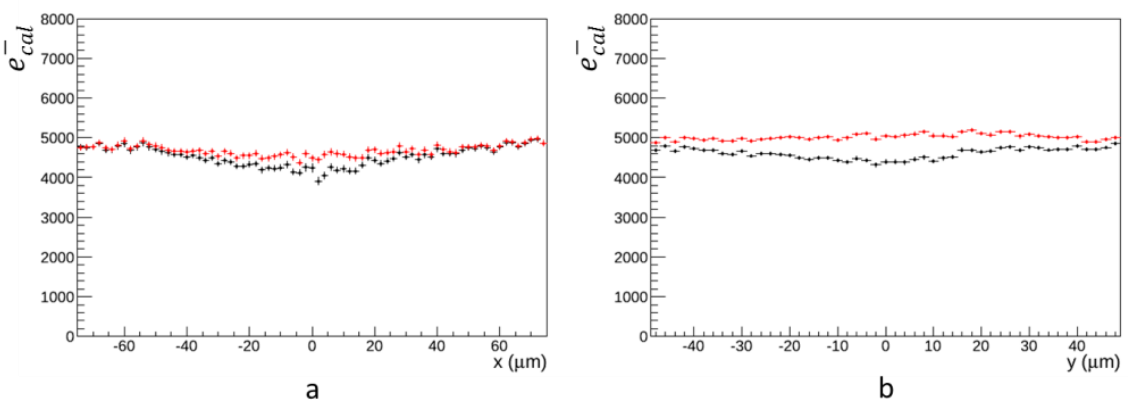

Figure 14: Mean value of the charge collected by the pointed-to pixel alone (black points) and that collected together with the nearer adjacent pixel (red points) on the same row (a) and on the same column (b). For further details see text.

Employing the same fiducial area cuts of Fig.12, we then studied the evolution of the chargecollection efficiency along a row or a column in a completely analogous way with what we did for Fig.5. The resulting histograms, requiring that the pointed-to pixel is always above

\footnotetext{
${ }^{3}$ This estimate comes from a simple model assuming a constant CCD value in the bulk. It is worth noting that CCD is about $170 \mu \mathrm{m}$ only, to be compared with a $500 \mu \mathrm{m}$ thickness.
} 
threshold, are shown in Fig.14. Also in this case, we observe a strong threshold bias, which is limiting the signal drop even across the pixel divide. Interestingly enough, sharing of charge up to about $50 \mu \mathrm{m}$ distance is clearly visible: the red points indeed match the black ones only asymptotically, at a distance $>50 \mu \mathrm{m}$. In principle, this effect could be attributed to a sizeable signal induction on nearby pixels and/or a relatively higher incidence of $\delta$-ray emission for high signals.

Once more, we made use of the scCVD data, with a threshold-cut of $15 \mathrm{ke}_{\text {cal }}^{-}$, to gauge the possible contribution of $\delta$-rays to extend the charge-sharing region. It turns out (Fig.15) that $\delta$ rays by themselves cannot explain the observed effect: the width of the charge-sharing region remains about the same as at low threshold (see Fig.5) and, even more, the double-hits are so few that their contribution is practically negligible (the red points almost always overlap the black ones). A sizeable signal induction, instead, could naturally increase the number of doublehits and widen the charge-sharing region.

Our conclusion is that our pCVD diamond shows important signal induction effects, which are responsible for spreading the signal over a wide region: very likely, two nearby pixels at least are always involved. New measurements with much more sensitive readout electronics are clearly required to further investigate this issue.
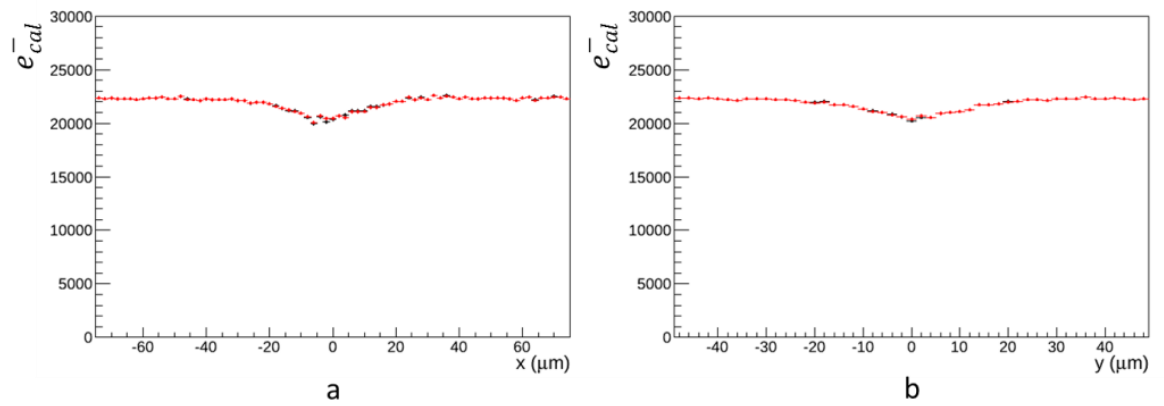

Figure 15: Same histograms as in Fig.12 for the scCVD detector with a $15 \mathrm{ke}_{\text {cal }}^{-}$threshold

In Fig. 16 we show the detection efficiency as a function of the track impact-point coordinates within the pointed-to pixel. As for Fig.6, the eight pixels around the pointed one are also allowed to contribute to the efficiency. As expected, the combined effect of threshold and signal induction heavily compromises the efficiency even at the pixel center. The detection efficiency averaged over the cell is very poor, $\varepsilon=0.6382 \pm 0.0005$.

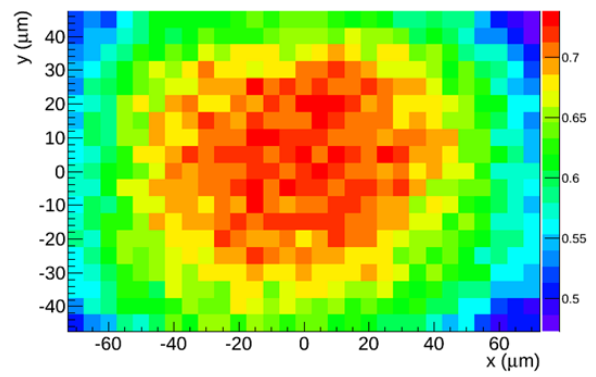

Figure 16: Detection efficiency as a function of the track impact-point within a pixel cell.

The efficiency evolution along a row or column, within the usual fiducial area of Fig.12 and asking for the efficiency of the pointed-to pixel alone (black points) or of it together with the nearer on the same row or column (red points), is reported in Fig.17. 

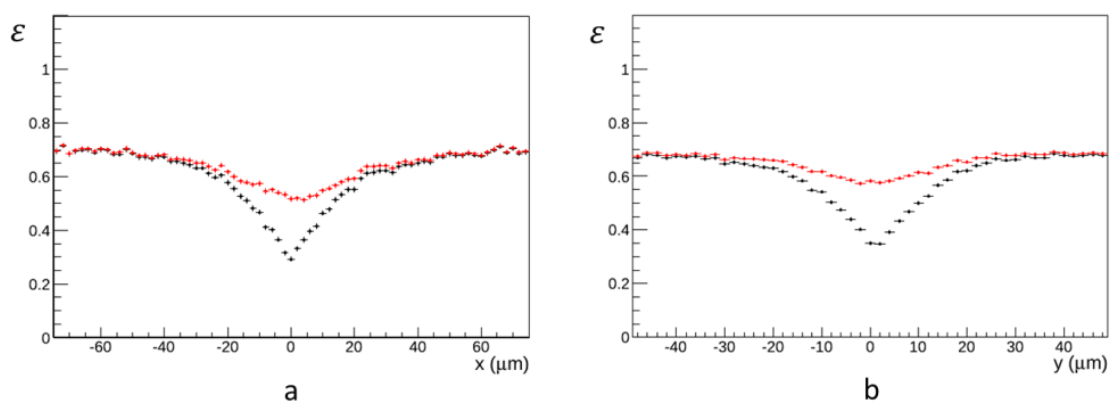

Figure 17: Detection efficiency evolution along a row (a) and a column (b); black points are for the efficiency of the pointed-to pixel alone and red points for the joint efficiency with the nearer adjacent pixel on the same row or column.

In agreement with the average collected charge evolution (see Fig.14) the efficiency of the pointed-to pixel starts to deteriorate already at about $50 \mu \mathrm{m}$ from the divide. The joint efficiency with the adjacent pixel does not compensate enough to recover the asymptotic value at the pixel center.

For the polycrystalline diamond detector too, the last measurement we performed concerns the charge-division feature and the achievable position resolution for double-hits. Fig. 18 shows the measured $x-\eta$ and $y-\eta$ correlations.
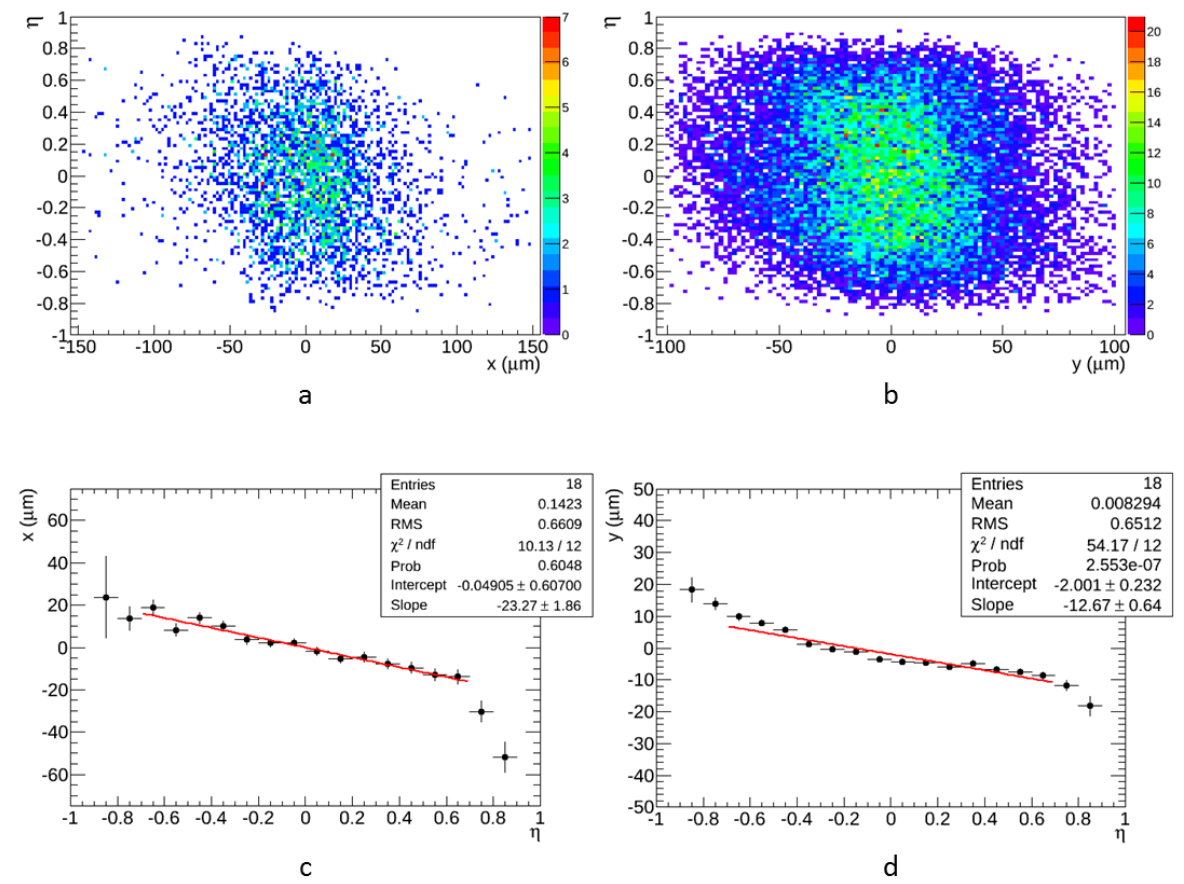

Figure 18: $x-\eta$ (a) and $y-\eta$ (b) correlation plots and their coordinate-averaged regressions. For further details see text.

The correlation extends beyond $100 \mu \mathrm{m}$ from the divide (see $x-\eta$ scatter plot) and, therefore, a proper interpolation should involve four adjacent hits at least in the best case. The tails of the $y$ $\eta$ correlation are cut out at $100 \mu \mathrm{m}$ by the physical dimension of the two adjacent pixels. This means that we shouldn't wonder if the correlation for double-hits alone turns out to be fuzzy 
and widely dispersed, and its fitted slope in $x-\eta$ differs from the one in $y-\eta$. In addition, the correlation should be diluted by the energetic $\delta$-rays, which are randomly emitted around the track direction.

On these bases, if we insist on this argument and calculate the coordinates using the fitted regressions, the resolution should be very poor. In fact, subtracting in quadrature the telescope track error from that of the residuals, as we did for the scCVD diamond, we obtain $\sigma_{x} \approx 33.7 \mu \mathrm{m}$ and $\sigma_{y} \approx 35.3 \mu \mathrm{m}$.

\section{Conclusions}

We have comparatively studied the tracking performance of a single-crystal and polycrystalline diamond pixel detector using a $120 \mathrm{GeV} / \mathrm{c}$ momentum beam of protons.

Our measurements demonstrate that the performance of a single-crystal diamond pixel-detector is comparable with that of the best available silicon detectors.

On the other hand, the study of a medium quality polycrystalline diamond (CCD $\approx 175 \mu \mathrm{m}$ ) with the same CMS readout-chip turns out to be challenging but, nonetheless, very instructive. A new chip, capable of operating at very low threshold (around $1 \mathrm{ke}^{-}$), is clearly required to reveal and exploit its hidden potential. Nonetheless, its behavior turns out to match pretty well what we would expect from a single-crystal diamond detector when operated at an equivalently high threshold. Complications due to a non-negligible signal induction are evident and constitute a further limitation for both the efficiency and the resolution.

\section{Acknowledgments}

We wish to thank the Fermilab Test Beam Facility personnel, and in particular Aria Soha, for the continuous support they provided us. In addition we would like to thank Bert Harrop (Princeton University) and Robert Stone (Rutgers University) for the assembly, especially pixellation and bump-bonding, of the detectors used in this study. This research was supported in part by the U.S. Department of Energy and the Italian Istituto Nazionale di Fisica Nucleare and Ministero della Ricerca Scientifica e Tecnologica.

\section{References}

[1] RD42 Collaboration, Development of diamond tracking detectors for high luminosity experiments at LHC, CERN-LHCC-2007-002, CERN-LHCC-RD-012

[2] H.C. Kästli et al, Design and Performance of the CMS Pixel Detector Readout Chip, Nucl.Instrum.Meth. A565 (2006) 188-194

[3] R. A. Rivera, M. Turqueti, L. Uplegger, A Telescope Using CMS PSI46 Pixels and the CAPTAN for Acquisition and Control over Gigabit Ethernet, 2009 IEEE Proc. Nuclear Science Symposium

[4] M. Turqueti et al, CAPTAN: A hardware architecture for integrated data acquisition, control, and analysis for detector development, FERMILAB-PUB-08-527-CD

[5] http://root.cern.ch/root/html/tutorials/fit/langaus.C.html

[6] R.J. Tapper, Diamond detectors in particle physics, Rept.Prog.Phys. 63 (2000) 1273-1316

[7] S. Ramo, Currents Induced by Electron Motion, Proceedings of the IRE 27 (9): 584-585 (1939) 
[8] W. Shockley, Currents to Conductors Induced by a Moving Point Charge, Journal of Applied Physics 9 (10): 635 (1938)

[9] RD42 Collaboration, Recent Advances in Diamond Detectors, arXiv:0810.3429 